\title{
Comparison of MWIR thermography and high-speed NIR thermography in a laser metal deposition (LMD) process
}

\author{
by S.J. Altenburg*, C. Maierhofer*, A. Straße*, A. Gumenyuk*
} *Bundesanstalt für Materialforschung und -prüfung, Unter den Eichen 87, 12205 Berlin, Germany,
Simon.Altenburg@bam.de

\begin{abstract}
Additive manufacturing (AM) offers a range of novel applications. However, the manufacturing process is complex and the production of defect-free parts with high reliability and durability is still a challenge. Thermography is a valuable tool for process surveillance, especially in metal AM processes. The high process temperatures allow one to use cameras usually operating in the visible spectral range. Here, we compare the results of measurements during the manufacturing process of a commercial laser metal deposition setup using a mid-wavelength-IR camera with those from a visual spectrum high-speed camera with band pass filter in the near-IR range.
\end{abstract}

\section{Introduction}

Additive manufacturing ( $\mathrm{AM}$ ) offers a range of novel applications, from the production of parts with a complex geometry that cannot be built by classical manufacturing in a single piece to highly individualized production on endcustomer demand. However, the manufacturing process is complex and the production of defect-free parts with high reliability and durability is still a challenge [1]. To overcome these issues, the manufacturing process is usually monitored by laser irradiation and camera based systems [2]. In addition to the surveillance of process parameters, different inprocess NDT techniques can be implemented. Thermography has been proven to be a valuable tool for melt pool control, especially in metal AM processes [1, 2]. It allows one to determine the temperature of the melt pool, its dimensions and dynamics as well as cooling rates. However, there are certain hurdles to be overcome to assure a reliable thermographic process surveillance in metal AM. First, the complexity of the emissivity (temperature, angle, aggregate state, and composition dependency) poses a challenge [3]. Second, depending on the specific AM process at hand (e.g., laser metal deposition (LMD), laser beam melting (LBM), wire arc AM (WAAM)), the accessibility of the process itself may be limited due to constraints defined by the production setup. For all laser based methods, laser safety has to be ensured. Despite these challenges, the high process temperatures of up to more than $2500 \mathrm{~K}$ (depending on the used material) allow one to use cameras usually operating in the visible spectral range to monitor the process, since a significant amount of thermal radiation is emitted in the visual spectrum (VIS) range. A calibration of a VIS camera for temperature measurement is possible (VIS thermography) [4]. To avoid disturbing influences from emissions of the welding plasma, a wavelength limitation of the detected radiation to a narrower band in the near infrared (NIR) is desirable and can be realized by a band pass filter.

Here, we present results of experiments for temperature monitoring during an LMD process, using currently available hardware, that were performed in order to gather experience for the choice of a process-optimized camera system. To this end, experiments using stationary cameras operating in the mid-wavelength infrared (MWIR) and the VIS range (limited by a narrow band pass filter in the NIR) were performed.

An approximate calibration of the cameras using the grey body approximation and the solidification temperature as input, was performed. It is shown that the VIS camera has a very limited measurable temperature range, compared to the MWIR camera. On the other hand, the MWIR camera detects strong reflections from the build plate and spacial inhomogeneities in the heated area that are most likely caused by inhomogeneities of the emissivity. The VIS camera did not show these effects. As a result, a camera operating in the short wavelength infrared (SWIR) will be tested next, in the assumption of a combination of the advantages of both tested measurement systems.

\section{Experimental details}

In this section, we describe the experimental setup.

\subsection{AM-setup}

The AM-setup, used for the experiments, is a commercial laser metal deposition cell of the manufacturer Trumpf, type TruLaser Cell 3000. It is coupled with a $16 \mathrm{~kW} \mathrm{Yb:YAG} \mathrm{TruDisk} 16002$ - disk laser (Trumpf) with a wavelength of $1030 \mathrm{~nm}$. The laser head can be moved in $\mathrm{x}$-, $\mathrm{y}$ - and $\mathrm{z}$-direction and tilted to both sides in a maximum angle of $135^{\circ}$.

The powder deposition into the laser beam is achieved with an integrated three-jet SO16-powder nozzle having a working distance of $16 \mathrm{~mm}$ measured from the nozzle edge. The three powder jets are arranged around the laser beam coaxially in $120^{\circ}$ angles, see figure 1 . The powder jets are concentrated in a spot of approximately $3.5 \mathrm{~mm}$ diameter at this distance. 
The optical head is equipped with a flexible collimation unit allowing to adjust the beam diameter in the range between 1 $\mathrm{mm}$ and approximately $3.8 \mathrm{~mm}$ at the working plane.
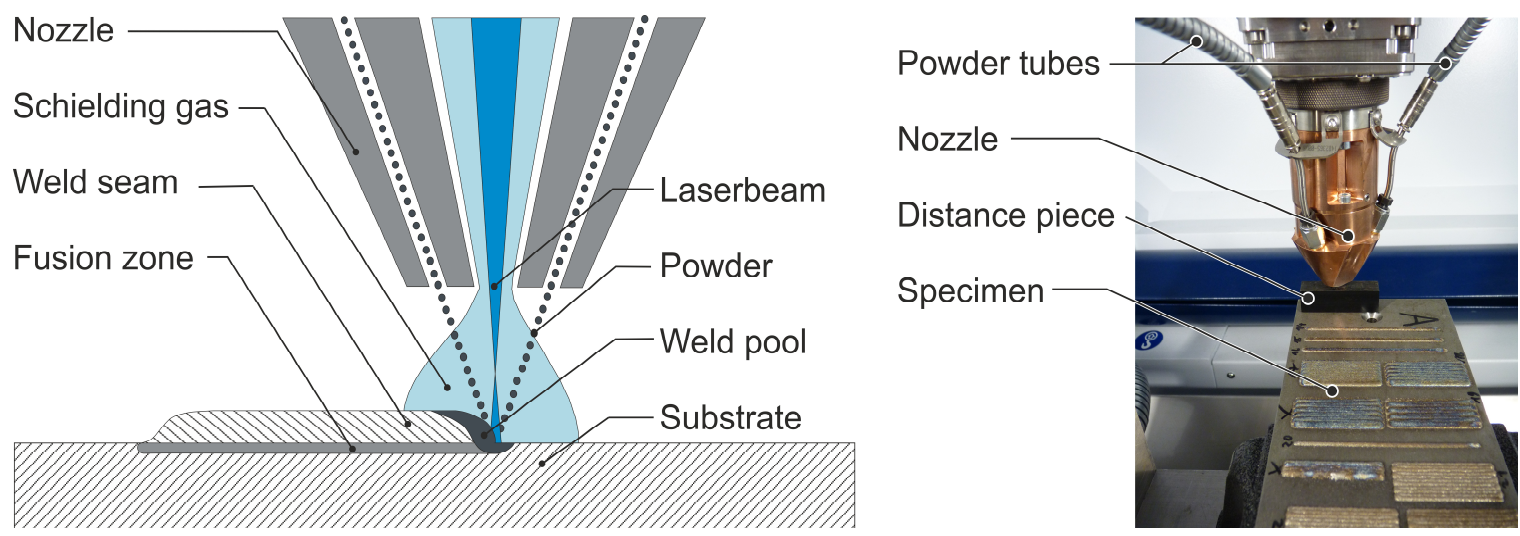

Fig. 1. Left: LMD-Process (schematic), right: Photograph of the SO16-nozzle with $16 \mathrm{~mm}$ distance piece.

\subsection{Thermographic setup}

Two cameras, operating in the MWIR and the VIS range, were used to monitor the thermal radiation emitted during the building process. The measurements were performed at different occasions in a comparable setup with similar process parameters. The MWIR (VIS) camera was mounted at a fixed position in the build chamber at a distance of $40 \mathrm{~cm}$ $(30 \mathrm{~cm})$ between frontmost lens and the focal point of the laser. The angle between optical axis of the camera and the surface normal of the base plate was approximately $60^{\circ}$. Photographs of the experimental setup are shown in figure 2 .

The used MWIR camera of type InfraTec ImagelR 8300 is sensitive in the spectral range between $2 \mu \mathrm{m}$ and $5 \mu \mathrm{m}$ (cooled InSb-focal-plane-array), is temperature calibrated in the range between $500^{\circ} \mathrm{C}$ and $1200^{\circ} \mathrm{C}$ (black body) and was operated at a frequency of $800 \mathrm{~Hz}$ in subframe-mode $(320 \times 156$ Pixels $)$ with a pixel resolution of $240 \mu \mathrm{m}$, resulting in an image width of $12.8 \mathrm{~cm}$. The integration time was set to $47 \mu \mathrm{s}$ and the bit resolution was 14 bit.

The VIS-camera of type Photron Fastcam SA4 (high speed camera with 32 GB internal memory) was equipped with a narrow band pass filter, limiting the detected light to a wavelength range between $807 \mathrm{~nm}$ and $817 \mathrm{~nm}$. It was operated at a frequency of $10 \mathrm{kHz}$ at a subframe resolution of $768 \times 240$ pixels with a pixel resolution of $48 \mu \mathrm{m}$ (image width: $3.7 \mathrm{~cm}$ ). An integration time of $100 \mu \mathrm{s}$ was set. To reduce the amount of data, the possible bit resolution of 12 bit was reduced to 8 bit.
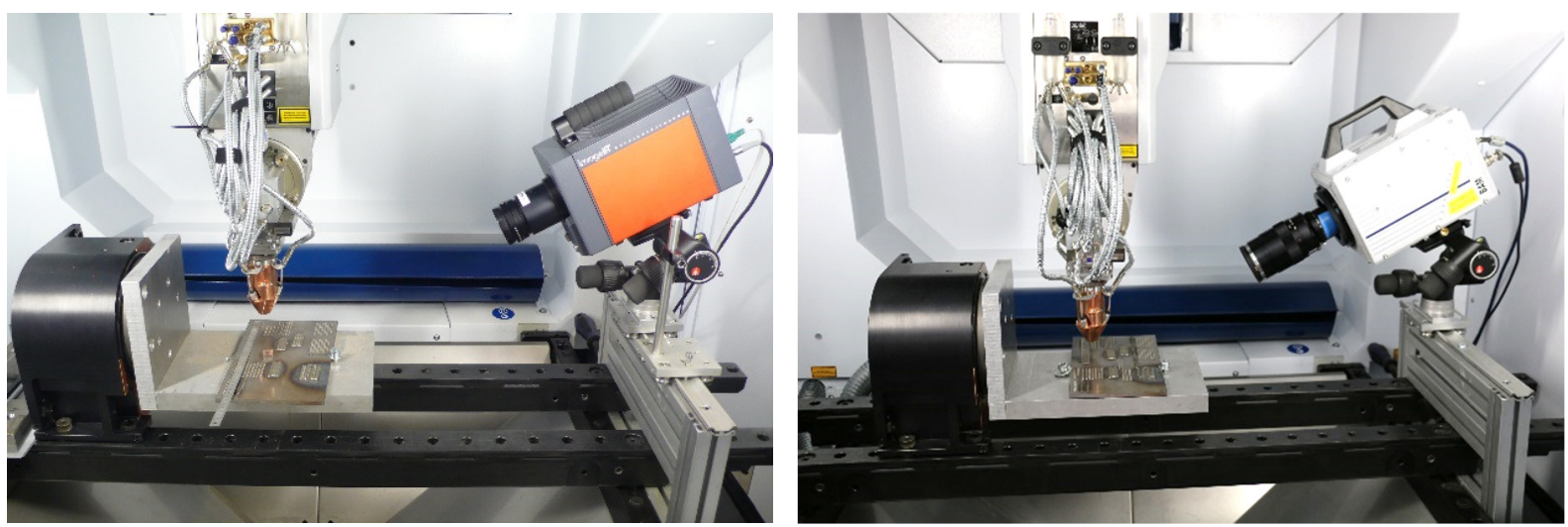

Fig. 2. Photographs of the experimental setup. Left: MWIR setup, right: VIS setup.

\subsection{Build parameters}

For the results presented here, the following build parameters were used: The build plate was made of carbon steel and the powder material was AISI 2205 duplex with an average diameter range from $53 \mu \mathrm{m}$ to $125 \mu \mathrm{m}$ and a mass flow density of $17 \mathrm{~g} / \mathrm{min}$. The laser power was set to $1.7 \mathrm{~kW}$ with a gaussian beam profile and a beam diameter of $3 \mathrm{~mm}$. The build speed was set to $13.3 \mathrm{~mm} / \mathrm{s}$. The powder carrier gas was helium with a flowrate of $4 \mathrm{l} / \mathrm{min}$ and the shielding gas was argon with a flowrate of $10 \mathrm{l} / \mathrm{min}$. The build geometry was a stack of single lines of $45 \mathrm{~mm}$ length consisting of up to 9 layers (3 layers) for the MWIR (VIS) camera, which were welded without pauses between the layers. 


\section{Experimental results and discussion}

Selected thermograms from the build process (building direction from right to left) for different layers recorded with the MWIR camera are shown in figure 3, together with a photograph of the built structure. Since the camera is calibrated assuming a perfect black body radiator, an assumption that is not met here, we do not give numbers on the intensity scale. Firstly, it can be seen that the size of the heated zone increases with the layer number, which can be explained by the decrease in heat dissipation to the build plate with an increase in part height. Secondly, also reflections from the base plate and shadowing of these reflections by other built structures on the build plate become visible in 3.b and more clearly in 3.c (compare to photograph in 3.d). Thirdly, the intensity distribution within the melt pool appears only in 3.a (layer 1) as one would expect (a maximum at the centre with a decay in all directions). In 3.b, a second maximum below the most intense one can be seen, that is probably caused by reflections. However, another local maximum can be seen on the right-hand side, whose origin is unclear. One probable cause is a local variation of the emissivity. In subfigure 3.c (upmost layer 9), the melt pool appears strongly inhomogeneous. In particular, a rim of increased intensity is observable around the melt pool, which is most likely rather caused by an increased emissivity in this region (slag formation, [5]) than by an actual temperature increase.

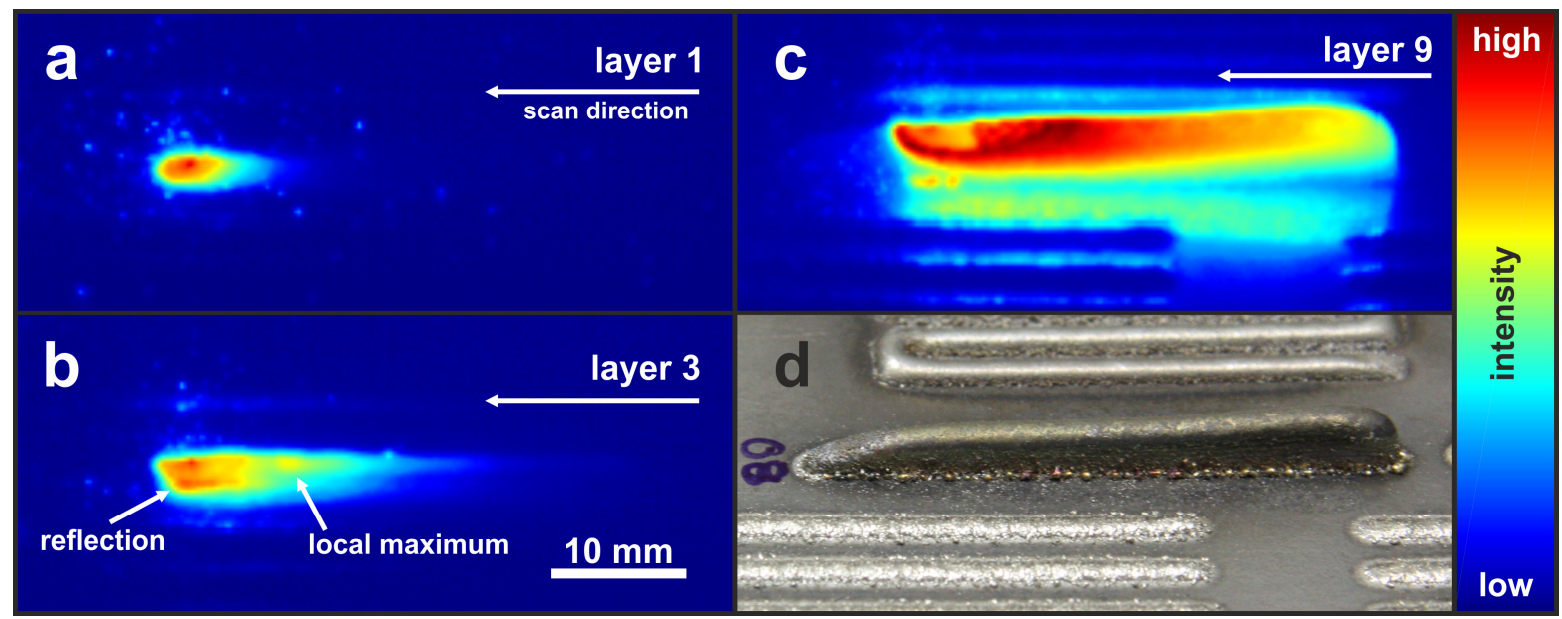

Fig. 3. Thermograms recorded with the MWIR camera (a to c) and photograph (d) of the built structure (centre, marked 08). a: First layer, the melt pool appears homogeneous with a well-defined hotspot at the centre. b: Third layer, a second hot spot marked "reflection" is observable as well as a local maximum. c: Ninth layer, a complicated intensity distribution is observable. There is a high intensity rim around the melt pool. Reflections from the build plate and shadowing by previously built structures can be seen (compare to $d$ ). $d$ : Photograph of the built structure from a similar angle. The structures shadowing in subfigure $c$ can be identified.

Figure 4 shows a comparison of a thermogram of the MWIR camera (same as figure 3.b) and the VIS camera, recorded during the build of the third layer. Comparing subfigures 4.a (MWIR) and 4.b (VIS) at the same geometric scale, the most remarkable difference is the different apparent size of the heated zone. This is mainly caused by the limited dynamic temperature range of the VIS camera in combination with the much larger intensity contrast between emitters of different temperatures in the NIR range than in the MWIR range. Using an approximate temperature calibration (see below), the temperature range of the VIS camera is limited to a range of $1000^{\circ} \mathrm{C}$ to $1700^{\circ} \mathrm{C}$, while the MWIR camera can measure from roughly $500^{\circ} \mathrm{C}$ to $2000^{\circ} \mathrm{C}$ in the current example, slightly exceeding the calibrated range (compare figure 5.a). Thus, the measurement of the VIS camera is limited to the hottest part of the process, which is limited to a smaller region in the thermogram. Contrary to the thermogram of the MWIR camera, there are no signs of reflections in the thermogram of the VIS camera. This is expected, since the reflectivity of unpolished metals strongly decreases with decreasing wavelength of the light. Additionally, possible reflections of lower intensity are cut off by the detection limit of the VIS camera. In the thermogram of the VIS camera, there is no sign of the additional local maximum on the right-hand side of the melt pool that can be seen in the MWIR image. Either the intensity of this structure is below the detection limit of the camera or the effect leading to this signature is not present at the wavelength of the VIS camera. Subfigure 4.c shows an enlarged view of subfigure 4.b. Due to the higher spacial resolution of the VIS camera a lot of details can be observed that are not visible in the MWIR thermogram. Firstly, we can see that there is a small region in the image that is overexposed (dark red), so the dynamic range of the camera is completely used and the highest intensity values are cut off. Similar to figure 3.c, there is a rim around the melt pool with an increased intensity. However, contrary to figure 3.c, the intensity value of this rim is well below the maximum intensity of the thermogram. From the time series of thermograms of the VIS camera (not shown here), it can be seen that various small low intensity spots can be attributed to powder particles that fly through the image, shadowing parts of the melt pool. Others of these spots appear to be swimming in the melt pool and dissolve after a certain time. An additional observation from the time series that is not visible in the single thermogram is a slight swirling of the melt pool. However, it is unclear whether these details will in fact be useful for purposes of process monitoring. 


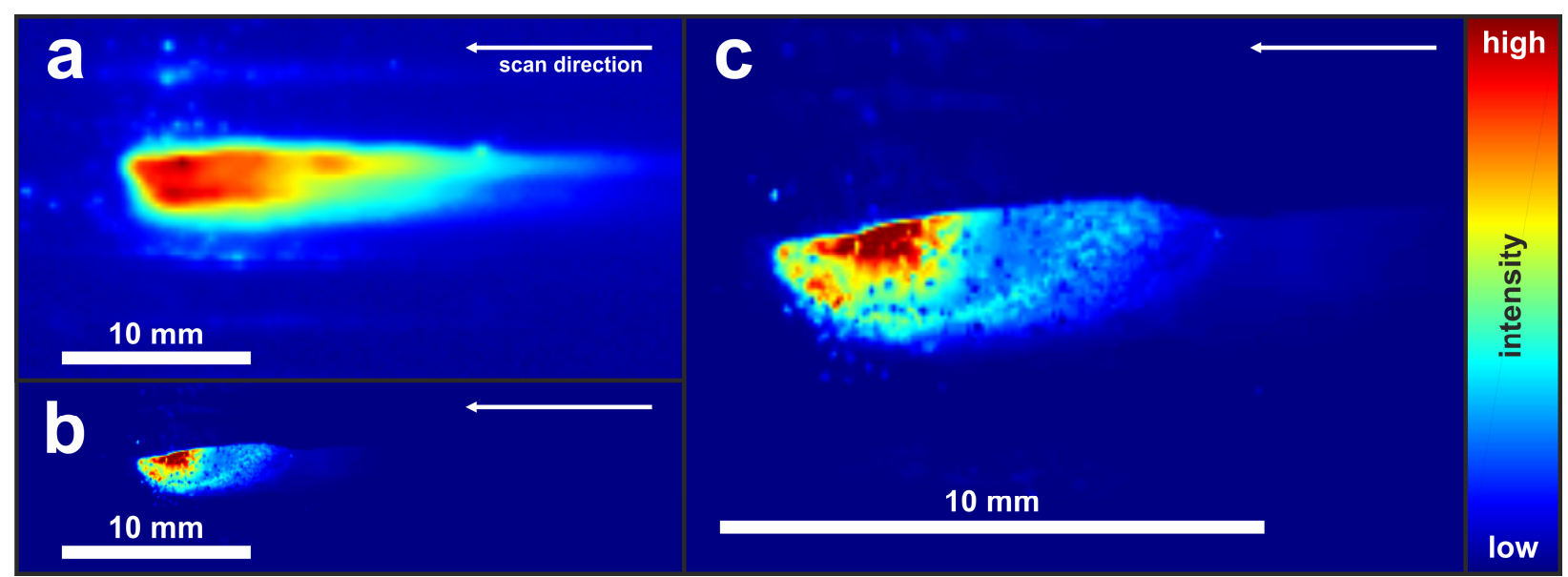

Fig. 4. Intensity maps, recorded during the build of the third layer of the stacks of lines. a: Recorded with the MWIR camera (same as figure 3.b). b/c: Recorded with the VIS-camera with NIR filter. b: Same scale as a, c: enlarged view. Mainly the melt pool is visible. Shadowing by powder particles and irregular structures within the melt pool can be observed as well as a slight rim of increased intensity around the melt pool.

Figure 5 shows the time evolution of the measured signals at single points in space that the laser passes with maximum intensity during the build of the first layer. Subfigure 5.a shows the signal of the MWIR camera for a single pixel. Note that the heating duration is approximately $0.23 \mathrm{~s}$, based on laser spot diameter and scan velocity. During heating, there is a shoulder in the apparent temperature, probably caused by the melting of the material. It is followed by a maximum (further heating of the melt), followed by a decrease of the apparent temperature, a plateau (caused by the solidification of the melt) and a further decrease. This general shape of the temperature development during the build has previously been reported [6] and allows one to determine an effective emissivity value by using the grey body approximation and adjusting the emissivity in such a way that the temperature of the solidification plateau equals the known solidification temperature of the used material. This procedure was performed assuming a solidification temperature of $1380^{\circ} \mathrm{C}$ (average of liquidus and solidus temperature) for AISI 2205 [7] and yielded an emissivity of $\epsilon=0.29$. Results of the correction are shown in subfigure 5.c. Note that the actual solidification temperature of the material is not known exactly, since the melted material is a mixture of the build plate material and the powder material. The results for the VIS camera are shown in subfigure 5.b. Here, the average of $5 \times 5$ pixels is shown (corresponding to the same area on the sample as one pixel in the case of the MWIR camera), that is additionally treated by a running average filter of 21 frames to reduce signal fluctuations. Like in the signal of the MWIR camera, the shoulders of melting and solidification can be observed, although the shoulder corresponding to the melting process is less pronounced. Since the data of the MWIR camera is already treated by a camera-internal radiometric model, a direct comparison of these results is not possible. To this end, using the grey body approximation and the assumed solidification temperature of $1380^{\circ} \mathrm{C}$, corrected temperature values for the signal of the VIS camera were calculated and are compared to those of the MWIR camera (corrected for the emissivity) in subfigure 5.c. Note that the curves were shifted along the time axis to allow for a direct comparison. Except for the cut-off temperature values outside the dynamic range of the VIS camera, the corrected temperature profiles match very well.
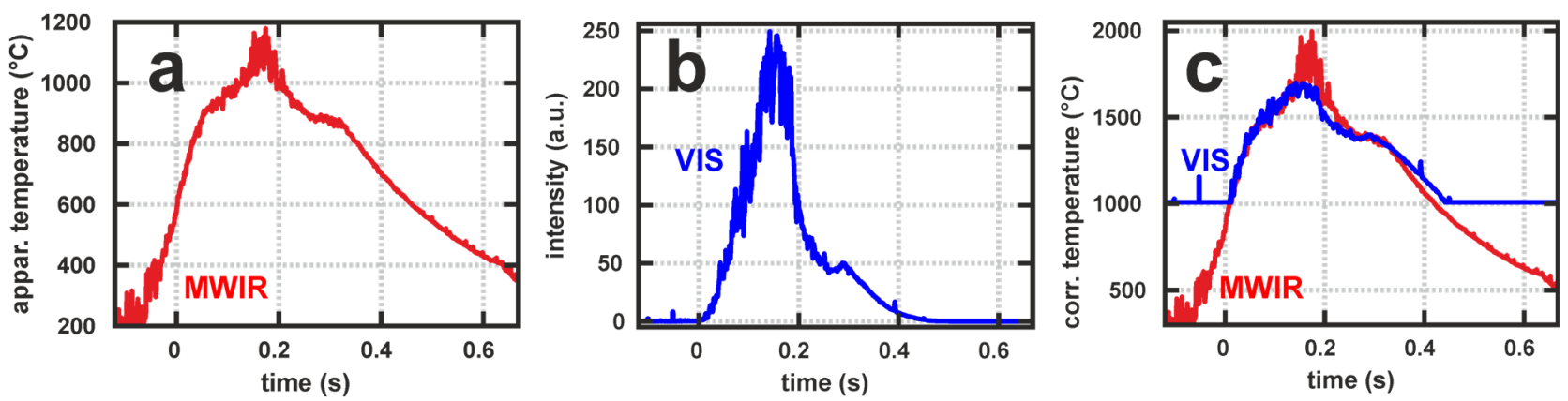

Fig. 5. Time evolution of the measured signal at a position that is passed by the laser in the first layer. The time axes were shifted to match. a: Measured with the MWIR camera (emissivity setting $\epsilon=1$ ), single pixel. b: Measured with the VIS camera and averaged over $5 \times 5$ pixel (to match the pixel size of the MWIR camera), using a running average filter of 21 frames. $c$ : Data of $a$ and $b$ after simple calibration procedure (see text). 


\section{Conclusion}

There are two main differences between the results of the MWIR and the VIS camera. Firstly, the limited dynamic range of the VIS camera leads to a limited measurable temperature range that might not be sufficient for process monitoring purposes. Contrary, the dynamic range of the MWIR camera leads to a large measurable temperature range. The second difference concerns disturbances due to reflections and variations of the emissivity. Both the reflection coefficient and the emissivity depend on the wavelength. The reflectivity of rough metal surfaces (e.g., the build plate) is much lower in the VIS range than in the MWIR range, as can be seen in the absence of reflections in the VIS data. The wavelength dependence of emissivity variations during the build process is so far unclear. However, the second local maximum that appears in the MWIR image in figure 4.a is not observable in the VIS camera image (figure 4.b), indicating lower emissivity variations in the case of the VIS camera. Additional experiments at a larger number of layers for the VIS camera will clarify this issue, since the emissivity variations appear to be strongly enhanced in this case for the MWIR cameras results (figure 3.c).

In essence, the VIS camera has the advantage of the absence of reflections that complicate the image analysis in the case of the MWIR camera. This advantage comes at the cost of a strongly reduced observable temperature range. As a next step, a camera operating in the SWIR range with a large dynamic range will be tested, in the assumption of a combination of the advantages of both camera systems tested here.

This research was funded by BAM within the focus area Material.

\section{REFERENCES}

[1] DebRoy T., Wei H. L., Zuback J. S., Mukherjee T., Elmer J. W., Milewski J. O., Beese A. M., Wilson-Heid A., De A., Zhang W., Additive Manufacturing of Metallic Components - Process, Structure and Properties, Progress in Materials Science. - Vol. 92pp. 112-224, 2018.

[2] Grasso M., Colosimo B. M., Process Defects and in Situ Monitoring Methods in Metal Powder Bed Fusion: A Review, Measurement Science and Technology. - Vol. 28, no 4, p. 044005, 2017.

[3] Schöpp H., Sperl A., Kozakov R., Gött G., Uhrlandt D., Wilhelm G., Temperature and Emissivity Determination of Liquid Steel S235, Journal of Physics D: Applied Physics. - Vol. 45, no 23, p. 235203, 2012.

[4] Yadroitsev I., Krakhmalev P., Yadroitsava I., Selective Laser Melting of Ti6al4v Alloy for Biomedical Applications: Temperature Monitoring and Microstructural Evolution, Journal of Alloys and Compounds. - Vol. 583pp. 404-409, 2014.

[5] Doubenskaia M., Pavlov M., Grigoriev S., Smurov I., Definition of Brightness Temperature and Restoration of True Temperature in Laser Cladding Using Infrared Camera, Surface and Coatings Technology. - Vol. 220pp. 244-247, 2013.

[6] Brandon L., Shawn M., P. W. E., Li M., Thermographic Measurements of the Commercial Laser Powder Bed Fusion Process at Nist, Rapid Prototyping Journal. - Vol. 22, no 5, pp. 778-787, 2016.

[7] Palmer T. A., Elmer J. W., Babu S. S., Observations of Ferrite/Austenite Transformations in the Heat Affected Zone of 2205 Duplex Stainless Steel Spot Welds Using Time Resolved X-Ray Diffraction, Materials Science and Engineering: A. - Vol. 374, no 1, pp. 307-321, 2004. 